\begin{tabular}{c} 
International Journal of Engineering \& Technology, 7 (4) (2018) 2733-2737 \\
International Journal of Engineering \& Technology \\
SPC \\
Website: $\begin{array}{c}\text { www.sciencepubco.com/index.php/IJET } \\
\text { doi: } 10.14419 / \text { ijet.v7it.16485 } \\
\text { Research paper }\end{array}$ \\
\hline
\end{tabular}

\title{
ECG signal diagnoses using intelligent systems
}

\author{
Raaed Faleh Hassan ${ }^{1}$ *, Sally Abdulmunem Shaker ${ }^{1}$ \\ ${ }^{1}$ Department of Medical Instrumentation Engineering Techniques, Middle Technical University, Iraq \\ *Corresponding author_E-mail: drraaed_alanbaki@eetc.mtu.edu.iq
}

\begin{abstract}
Accurate diagnosis of arrhythmias plays a crucial role in saving the lives of many heart patients. The aim of this research is to find the more efficient method to diagnosis electrocardiogram (ECG) diseases. This work presents the use of Backpropagation neural network (BPNN) and fuzzy logic for automatic detection of cardiac arrhythmias based on analysis of the ECG. These a more valuable tool used to classify ECG signals in cardiac patients. Data collected from physioBank ATM. The analysis of the ECG signal is performed in MATLAB environment. In BPNN the results appear that the only two misclassifications happened to result in an accuracy of $90.4 \%$. while in fuzzy inference system the results appear that the classification accuracy is $100 \%$.
\end{abstract}

Keywords: ECG; Backpropagation; Fuzzy; MATLAB; Cardiovascular Diseases

\section{Introduction}

The electrocardiogram is the registration of electrical activity of the cardiac and its effective method for a diagnosis of heart disease. The ECG signal includes P-QRS-T waves, each wave reflects a certain electrical activity of the heart, $\mathrm{P}$ wave represents atrial depolarization, QRS complex represents ventricular depolarization and $\mathrm{T}$ wave represents ventricular repolarization [1]. The practitioners then explicate the shapes of those waves and complexes, they compute the parameters to decide if the ECG appears signs of presence heart disease or not. The parameters are the amplitude and the duration of each wave, such as R-R period, Q-T, P$\mathrm{R}$ period and S-T segment [2].

Electrocardiography is having a critical role in diagnosing the situation of the heart. It supplies useful information about the functional parts of the cardiac and cardiovascular system. untimely detection of cardiac diseases/abnormalities can save lives and improve the quality of living through of suitable therapy. For efficient diagnostics, the research of ECG shape and cardiac rate variability signal may execute through several hours. Therefore, the number of the data is massive, the study is boring and prolonged. Naturally, the opportunity of the analyst absents or misreading is high. so, computer-based analysis and categorizing of heart diseases can be very useful in diagnostics [1].

Commonly, the ECG analysis can be performed through [4] steps: Acquiring step, in which the ECG signal is acquired as a result of leads voltage differences. According to its dynamic range which is between $(0.5-100) \mathrm{Hz}$, the ECG signal suffers from the baseline wander noise and the power line interference, this shows the need for a step to remove these noises namely de-noising step. The third step in the ECG analysis system is the feature extraction of the clean ECG signal, while the fourth step is the diagnosis of the ECG signal based on its features [3].

Because the behavioral model of non-stationary electric activity of the heart and for provide the most accurate results. Will use artificial neural networks (ANN) and fuzzy sets for ECG diagnosis. These techniques will be able to adapt to the behavioral of ECG signal.
Neural networks are in consideration because of their selfacclimatization and strengths. In additional words, neural networks are a non-linear map between the inputs and the actual outputs. Its information is stocked in the weighted net among nodes. The algorithm of fuzzy sets is others widespread solution for ECG analyzing and diagnosing due to it employ smooth variables with membership functions for the medical conclusion. The conventional fuzzy classifier usually depends on the prior information and experience of field experts. It varies from neural networks as non-linear mapping [4].

Through past little years, a lot of researchers advanced various techniques and algorithms to categorize the ECG signals. In paper [5] Processing method use Wavelet and Fuzzy Logic Control, feature vector extracted are normalized energy and entropy, The target of improving methodology is to classify ECG signal to normal beats and abnormal beats, this work investigated by employ the MIT-BIH Arrhythmia Database. With the classification accuracy of $97 \%$.

In [6] the author employs a various algorithm such Discrete Wavelet Transform (DWT), Adaptive Least Mean Square (ALMS) and Support Vector Machine (SVM) for QRS complex detection and ST-segment detection. Then classify to normal and arrhythmia using SVM. using MIT-BIH arrhythmia database for investigation this work with accuracy for detection is $98.65 \%$. In [7] the authors use Wavelet and backpropagation Neural Network, aim to diagnose three types of heart diseases with accuracy $92 \%$.

In [8] Processing method uses a wavelet transform and probabilistic neural network, Feature classification are 8 heartbeat conditions with Classification accuracy is $92.75 \%$. In [9] use DWT (db3) for R peaks detection, Principal Component Analysis (PCA) on detail coefficients for statistical feature extraction and Adaptive Neuro-Fuzzy Inference System for classification; four classes of ECG The proposed system give an overall classification accuracy of $97.75 \%$.

In this paper, 11 input features vectors which are: $(\mathrm{P}, \mathrm{Q}, \mathrm{R}, \mathrm{S}$ and T) amplitude, and (PR, RR1, RR2 and QRS) interval, ST deviation, and beat per minute have been considered. Back-propagation neural network(BPNN) and fuzzy logic are employed for ECG signal classification into normal and different 9 types of cardio- 
vascular diseases. Table 1 clarify different types of diseases these will be discussed in this paper [10].

Table 1: Show the Diseases with Characteristics Feature.

\begin{tabular}{|c|c|c|}
\hline NO. & Name of disease & characteristics feature \\
\hline 1 & $\begin{array}{l}\text { 1st degree heart } \\
\text { block }\end{array}$ & The PR-interval is prolonged over $0.2 \mathrm{~s}$ \\
\hline 2 & Atrial premature & $\mathrm{R} \_\mathrm{R}$ interval is variable \\
\hline 3 & Atrial fibrillation & $\begin{array}{l}\text { Heart rate (100-160) BPM, } \mathrm{P} \text { wave is very } \\
\text { small in amplitude or no } \mathrm{P} \text { wave and irreg- } \\
\text { ular rhythm. }\end{array}$ \\
\hline 4 & $\begin{array}{l}\text { Bundle-branch } \\
\text { block (BBB) }\end{array}$ & QRS interval is more than $0.12 \mathrm{sec}$ \\
\hline 5 & $\begin{array}{l}\text { Premature ventricu- } \\
\text { lar contraction } \\
\text { (PVC) }\end{array}$ & $\begin{array}{l}\text { One QRS interval is more than } 0.12 \mathrm{sec} \\
\text { and irregular rhythm. }\end{array}$ \\
\hline 6 & $\begin{array}{l}\text { Supraventricular } \\
\text { tachycardia (SVTA) }\end{array}$ & $\begin{array}{l}\text { No } P \text { wave, narrow } Q R S \text { complex and } \\
\text { heart rate }(140-250) \text { BPM. }\end{array}$ \\
\hline 7 & $\begin{array}{l}\text { Ventricular tachy- } \\
\text { cardia (VT) }\end{array}$ & $\begin{array}{l}\text { Wide QRS complex and heart rate more } \\
\text { than } 120 \text { BPM. }\end{array}$ \\
\hline 8 & $\begin{array}{l}\text { Myocardial Infrac- } \\
\text { tion(MI) }\end{array}$ & ST segment and $\mathrm{T}$ wave changes \\
\hline 9 & $\begin{array}{l}\text { Ventricle Hypertro- } \\
\text { phy }\end{array}$ & $\begin{array}{l}(\mathrm{R}(\mathrm{I})+\mathrm{S}(\mathrm{III})) \text { wave amplitude }>2.5 \mathrm{mv} \text {, } \\
\mathrm{R} \text { amplitude in lead } \mathrm{v} 1>0.7 \mathrm{mv} \text {. }\end{array}$ \\
\hline
\end{tabular}

\section{Classifier structure}

Fig. 1 shows a practical ECG classifier which is, in general, include two main blocks: the first block is ECG Parameters employed to extracted different features of the ECG signal. These features contain amplitude and interval of each wave and complex in ECG signal. Then these features exported as input to second function block for ECG classifier block which is used to diagnosis ECG diseases. ECG databases are collected from the MIT-BIH arrhythmia database and PTB database [11].

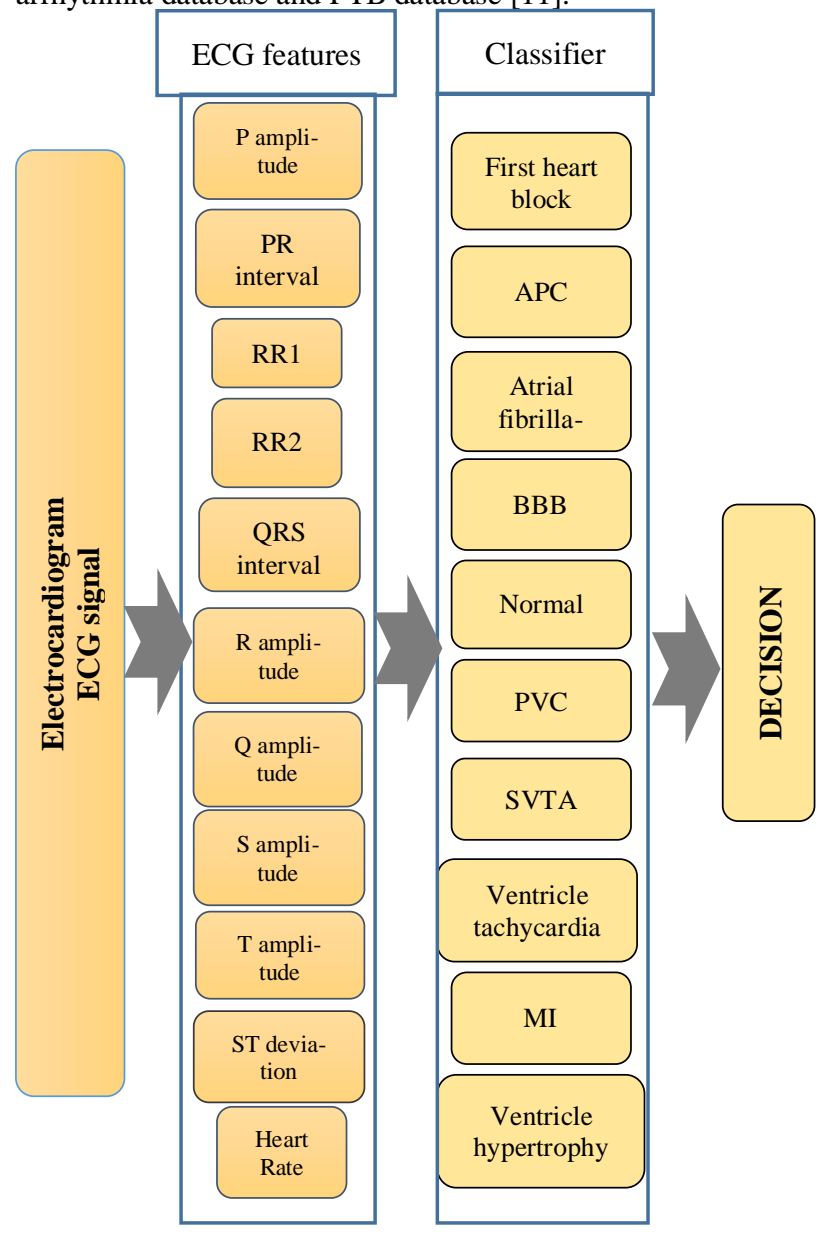

Fig. 1: The Structure of an Intelligent ECG Classifier In this paper two methods are employed to diagnosis cardiovascular diseases and classify ECG signal, the methods are:

\subsection{Artificial neural network classifier}

Artificial Neural Networks (ANNs) are mathematical models inspired by biological neurons. A traditional ANN contains three layers (an input, a hidden and an output) layer. features extracted from the ECG signal is exported to the input layer. output layer gives the diagnostic results of the input features for ECG signal. To get the proper classification performance, the choose of hidden nodes is very significant. any hidden unit in the hidden layer of ANN calculates the weighted total of its input to outline its scalar a net activation [12], [13].

The BPNN algorithm propagates back the error between the required output and the actual output across the network. After giving inputs to the input layer, the output layer of the network is then compared with a given target output and the error of all output unit are computed. This error is propagated back, and a closedloop control system is created [14]. In the training phase, weights in a network is changed to adapt to a target pattern to improve the network performance. The path in which these weights are changed is defined by the learning rule. The most common rules are the popularization of the Mean Square Error (MSE) rule. The proposed

Structure of the neural network in this paper consisted of 11 input nodes, one hidden layer with 10 units, and 10 units in an output layer, a number of hidden layers and units for each hidden layer was selected through trial and error as seen in figure 2 .

The characteristics extracted from the ECG signal are trained by applying the BPNN algorithm. 80 ECG signal of healthy and unhealthy persons for ECG signal was used in the learning phase, testing phase was executed with 21 ECG signal including healthy and unhealthy data and the results showed high accuracy.

The best performance of a scaled conjugate gradient backpropagation training function with 24 iterations where the present error is 0 . The test phase is performed by one input pass (each input is 11 features) using the weight obtained in the learning phase. Then calculate the output which as either normal, BBB, APC, PVC, $1^{\text {st }}$ block heart, Atrial fibrillation, SVTA, ventricle tachycardia, MI, or Ventricle Hypertrophy based on the expected values in the target data.

The activation function of the output layer is a log-sigmoid transfer function. One hot encoding is considered for interpreting the max output in each column as [1] and the rest as 0 [15], [16].

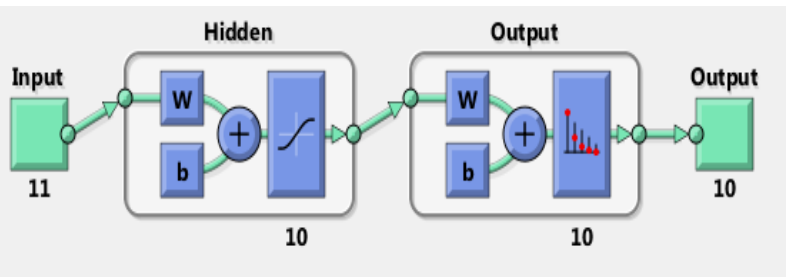

Fig. 2: Block Diagram of Neural Network.

\subsection{Fuzzy classifier}

Fuzzy logic might be calculated a continuation of the binary logic theory which has not demand crisp description and distinction, it's also very strong because it interprets the heuristics from human experts into perceptible quantitative data and therefore into the beneficial appreciation.

The fuzzy classifier system is a machine learning system that uses linguistic rules and fuzzy sets in its performance and a developmental algorithm for rule detection. Fuzzy logic is a comparatively youth theory. The main feature of this theory is that it permits the natural depiction, in the linguistic expression, of problems that must be fit instead than in terms of relationships between accurate numerical values. Fuzzy inference is the operation of formulating the mapping from a given input to an output to utilize fuzzy logic. The procedure of fuzzy inference involves membership functions, fuzzy logic operators and if-then rules. There are two kinds of fuzzy inference systems which can be executed in the Fuzzy Logic 
Toolbox: Mamdani-type and Sugeno-type. Mamdani's fuzzy inference method is the most generally seen the fuzzy methodology and it expects the output membership functions to be fuzzy sets [5]. Fuzzy Inference system (FIS) has been developed in this work using MATLAB' s fuzzy logic toolbox. The suggest FIS is seen in fig. 3 and is formed of the following:

- Ten inputs membership represent by the features: (P, R, S and T) amplitude, (PR, RR1, RR2 and QRS) interval and ST deviation, and beat per minute.

- One output membership descriptors for the diseased cases contain ten components (either normal, BBB, APC, PVC, 1st block heart, Atrial fibrillation, SVTA, ventricle tachycardia, MI, or Ventricle Hypertrophy).

- A set of 34 rules shown in Table 2 which interface different degrees of memberships to outputs.

A Fuzzy Mamdani algorithm was used to get appropriate boundaries between these classes. When input features are amplitude the boundary values are then used as normal and abnormal when input features are an interval, boundary values are then used as low, disappear, normal, and high. The trapezoidal membership function is employed as appear in figure 4. The output of the FIS represents the different type of disease, the centroid defuzzification method is used, the triangular membership function is employed in output. The $\mathrm{x}$-axis in each curve corresponds to the input value for each input membership function. The y-axis is from 0 to 1 represent the total probabilistic space [4], [17].

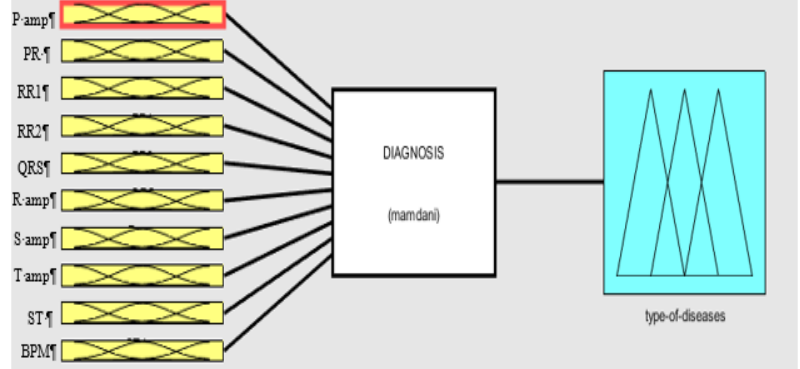

Fig. 3: The FIS Structure for the ECG Classifier.

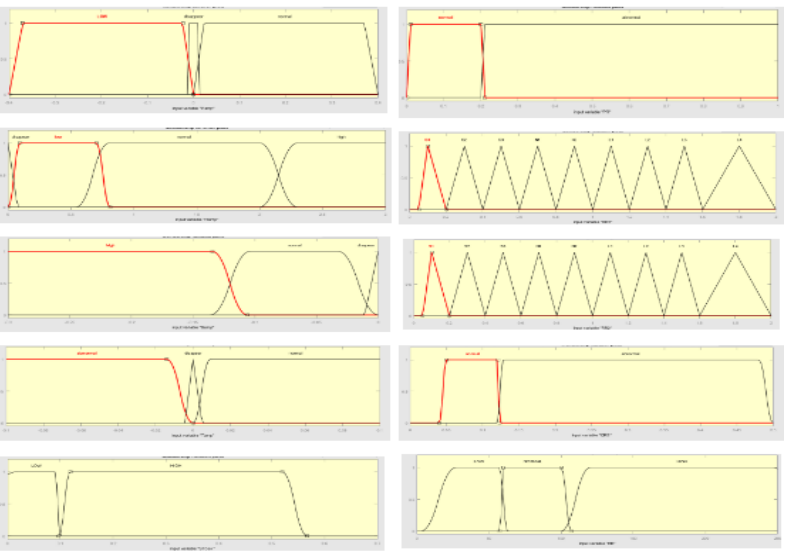

Fig. 4: The Fuzzy Input.

Table 2: Fuzzy Rules for ECG Diseases Diagnosis

\begin{tabular}{|c|c|c|c|c|c|c|c|c|c|c|c|}
\hline $\begin{array}{l}\text { Rule } \\
\text { number }\end{array}$ & Linguistic & puts & & & & & & & & & Linguistic output \\
\hline 1 & P mv & PR sec & RR1 sec & RR2 sec & QRS sec & $\begin{array}{l}\text { R mv } \\
\text { Normal }\end{array}$ & S mv & $\mathrm{T} \mathrm{mv}$ & ST deviation & BPM & $\begin{array}{l}\text { output } \\
\text { stheart block }\end{array}$ \\
\hline 2 & normal & abnormal & S1 & Not S1 & normal & --- & --- & --- & low & --- & APC \\
\hline 3 & normal & abnormal & S2 & Not S2 & normal & --- & --- & --- & low & --- & APC \\
\hline 4 & normal & abnormal & S3 & Not S3 & normal & --- & --- & --- & low & --- & APC \\
\hline 5 & normal & abnormal & $\mathrm{N} 1$ & Not N1 & normal & --- & --- & --- & low & --- & APC \\
\hline 6 & normal & abnormal & $\mathrm{N} 2$ & Not N2 & normal & --- & --- & --- & low & --- & APC \\
\hline 7 & normal & abnormal & L1 & Not L1 & normal & --- & --- & --- & low & --- & APC \\
\hline 8 & normal & abnormal & L2 & Not L2 & normal & --- & --- & --- & low & --- & APC \\
\hline 9 & normal & abnormal & L3 & Not L3 & normal & --- & --- & --- & Low & --- & APC \\
\hline 10 & normal & abnormal & L4 & Not L4 & normal & --- & --- & --- & Low & --- & APC \\
\hline 11 & disappear & --- & S1 & Not S1 & normal & Normal & --- & --- & Low & --- & Atrial fibrillation \\
\hline 12 & Disappear & --- & S2 & Not S2 & normal & Normal & --- & --- & Low & --- & Atrial fibrillation \\
\hline 13 & Disappear & --- & S3 & Not S3 & normal & Normal & --- & --- & Low & --- & Atrial fibrillation \\
\hline 14 & Disappear & --- & N1 & Not N1 & normal & Normal & --- & --- & Low & --- & Atrial fibrillation \\
\hline 15 & Disappear & --- & $\mathrm{N} 2$ & Not N2 & normal & Normal & --- & -- & Low & --- & Atrial fibrillation \\
\hline 16 & Disappear & --- & L1 & Not L1 & normal & Normal & --- & --- & Low & --- & Atrial fibrillation \\
\hline 17 & Disappear & --- & L2 & Not L2 & normal & Normal & --- & --- & Low & --- & Atrial fibrillation \\
\hline 18 & Disappear & --- & L3 & Not L3 & normal & Normal & --- & --- & Low & --- & Atrial fibrillation \\
\hline 19 & Disappear & --- & L4 & Not L4 & normal & Normal & --- & --- & Low & --- & Atrial fibrillation \\
\hline 20 & normal & abnormal & --- & --- & abnormal & Normal & --- & normal & Low & --- & BBB \\
\hline 21 & normal & normal & --- & --- & normal & Normal & --- & normal & Low & normal & Normal \\
\hline 22 & --- & --- & S1 & Not S1 & abnormal & Normal & --- & normal & Low & --- & PVC \\
\hline 23 & --- & --- & S2 & Not S2 & abnormal & Normal & --- & normal & Low & --- & $\mathrm{PVC}$ \\
\hline 24 & --- & --- & S3 & Not S3 & abnormal & Normal & --- & normal & Low & --- & PVC \\
\hline 25 & --- & --- & $\mathrm{N} 1$ & Not N1 & abnormal & Normal & --- & normal & Low & --- & PVC \\
\hline 26 & --- & --- & $\mathrm{N} 2$ & Not N2 & abnormal & Normal & --- & normal & Low & --- & PVC \\
\hline 27 & --- & --- & L1 & Not L1 & abnormal & Normal & --- & normal & Low & --- & $\mathrm{PVC}$ \\
\hline 28 & --- & --- & L2 & Not L2 & abnormal & normal & --- & normal & Low & --- & PVC \\
\hline 29 & --- & --- & L3 & Not L3 & abnormal & normal & --- & normal & low & --- & PVC \\
\hline 30 & --- & --- & L4 & Not L4 & abnormal & normal & --- & normal & low & --- & PVC \\
\hline 31 & disappear & --- & --- & --- & normal & --- & --- & --- & low & high & SVTA \\
\hline 32 & --- & --- & --- & --- & abnormal & --- & --- & --- & low & high & VT \\
\hline 33 & --- & --- & --- & --- & --- & --- & --- & abnormal & high & --- & MI \\
\hline 34 & --- & --- & --- & --- & --- & high & high & --- & Low & --- & V. hypertrophy \\
\hline
\end{tabular}




\section{Result and discussion}

The features extracted from the ECG signal for various cardiac diseases shown are in Table 3 and the features are parted into 11 parts. This feature will be input for a neural network and fuzzy. The classification result divided into two ways:

\subsection{Backpropagation neural network result}

Table 4 shows the output matrix for ECG diseases classification, the prediction made by the backpropagation neural network first 80 datasets were trained and then applied 21 datasets for the test phase. Eight classes are classified perfectly but two signals are error classified.

\subsection{Fuzzy result}

Figure 5 show membership function for fuzzy output and linguistic variables for each disease. Based on figure 5 we conclude all results in table 5 are correctly classified. From this results show the overall ECG diseases classification accuracy by BPNN is $90.4 \%$ and the overall ECG diseases classification accuracy by Fuzzy Inference system is $100 \%$.

Table 3: Extracted Features for ECG Signals

\begin{tabular}{|c|c|c|c|c|c|c|c|c|c|c|c|c|}
\hline Diseases & Record & P mv & PR sec & RR1 sec & RR2 $\mathrm{sec}$ & QRS sec & $\mathrm{R} \mathrm{mv}$ & $\mathrm{Qmv}$ & $\mathrm{S} \mathrm{mv}$ & $\mathrm{T} \mathrm{mv}$ & ST deviation & BPM \\
\hline $\begin{array}{l}\text { 1st heart } \\
\text { block }\end{array}$ & $231 \mathrm{~m}$ & 0.0516 & 0.2707 & 0.8889 & 0.8861 & 0.1194 & 0.8374 & 0 & 0 & 0 & 0 & 68 \\
\hline APC & $108 \mathrm{~m}$ & 0.1462 & 0.2714 & 0.9194 & 0.5222 & 0.0951 & 0.7109 & -0.0669 & -0.4736 & 0 & 0 & 57 \\
\hline APC & $112 \mathrm{~m}$ & 0.0687 & 0.212 & 0.9639 & 0.6306 & 0.0794 & 0.8232 & -0.0335 & -0.4123 & 0 & 0 & 74 \\
\hline APC & $114 \mathrm{~m}$ & 0.0846 & 0.259 & 0.7111 & 0.2694 & 0.0998 & 0.1903 & -0.1098 & -0.2172 & 0 & 0 & 102 \\
\hline $\mathrm{AF}$ & $202 \mathrm{~m}$ & 0.0089 & 0 & 0.7833 & 0.4361 & 0.1248 & 1.5948 & -0.1055 & -0.2134 & 0 & 0 & 139 \\
\hline $\mathrm{AF}$ & $203 \mathrm{~m}$ & 0 & 0 & 0.7306 & 0.4056 & 0.1096 & 1.0979 & -0.1081 & -0.2972 & 0 & 0 & 121 \\
\hline BBB & $231 \mathrm{~m}$ & 0.0516 & 0.2707 & 0.8889 & 0.8861 & 0.1438 & 0.8374 & -0.2491 & -0.0771 & 0.0247 & 0 & 51 \\
\hline BBB & $118 \mathrm{~m}$ & 0.1569 & 0.2944 & 0.8444 & 0.8472 & 0.1283 & 1.6007 & -0.0783 & -1.0729 & 0.1808 & 0 & 68 \\
\hline Normal & $106 \mathrm{~m}$ & 0.1604 & 0.1351 & 0.9194 & 0.9278 & 0.0494 & 2.2017 & -0.2836 & -0.5289 & 0.4161 & 0 & 62 \\
\hline Normal & $112 \mathrm{~m}$ & 0.0758 & 0.2 & 0.6889 & 0.6944 & 0.0994 & 0.7732 & -0.0536 & -0.2707 & 0.1338 & 0 & 79 \\
\hline Normal & $115 \mathrm{~m}$ & 0.016 & 0.2 & 0.9806 & 0.975 & 0.0997 & 1.8527 & -0.1562 & -0.7532 & 0.1178 & 0 & 62 \\
\hline PVC & $208 \mathrm{~m}$ & 0.0979 & 0.2548 & 1.0194 & 0.5694 & 0.2054 & 1.554 & -0.2619 & -0.4103 & 0.2791 & 0 & 114 \\
\hline PVC & $200 \mathrm{~m}$ & 0.0717 & 0.3542 & 0.8028 & 0.65 & 0.3472 & 0.8419 & -0.302 & -0.2162 & 0.1642 & 0 & 92 \\
\hline PVC & $210 \mathrm{~m}$ & 0.0317 & 0.3662 & 1.5444 & 0.5583 & 0.2544 & 1.1464 & -0.1004 & -0.2229 & 0.0676 & 0 & 103 \\
\hline SVTA & $207 \mathrm{~m}$ & 0 & 0 & 0.4056 & 0.4028 & 0.0848 & 0.4124 & -0.379 & 0 & 0 & 0 & 133 \\
\hline VT & $210 \mathrm{~m}$ & 0 & 0 & 0.5639 & 0.5833 & 0.222 & 0.0697 & 0 & 0 & 0 & 0 & 188 \\
\hline VT & $213 \mathrm{~m}$ & 0 & 0 & 0.56 & 0.5639 & 0.1526 & 2.2458 & 0 & 0 & 0 & 0 & 153 \\
\hline MI & s00591rem & 0 & 0 & 0 & 0 & 0.0506 & 0.8716 & -0.4441 & -0.1374 & 0.0486 & 0.1105 & 76 \\
\hline MI & s0062lrem & 0 & 0 & 0 & 0 & 0.0834 & 0.6414 & -0.2367 & -0.1552 & -0.0183 & 0 & 65 \\
\hline MI & s00691rem & 0 & 0 & 0 & 0 & 0.0844 & 0.6729 & -0.2263 & -0.1463 & -0.0333 & 0 & 59 \\
\hline $\begin{array}{l}\text { V. hypertro- } \\
\text { phy }\end{array}$ & s0438_rem & 0 & 0 & 0 & 0 & 0 & 2.5 & -0.1557 & -1.3 & 0 & 0 & 0 \\
\hline
\end{tabular}

Table 4: The Output of Back-Propagation Neural Network

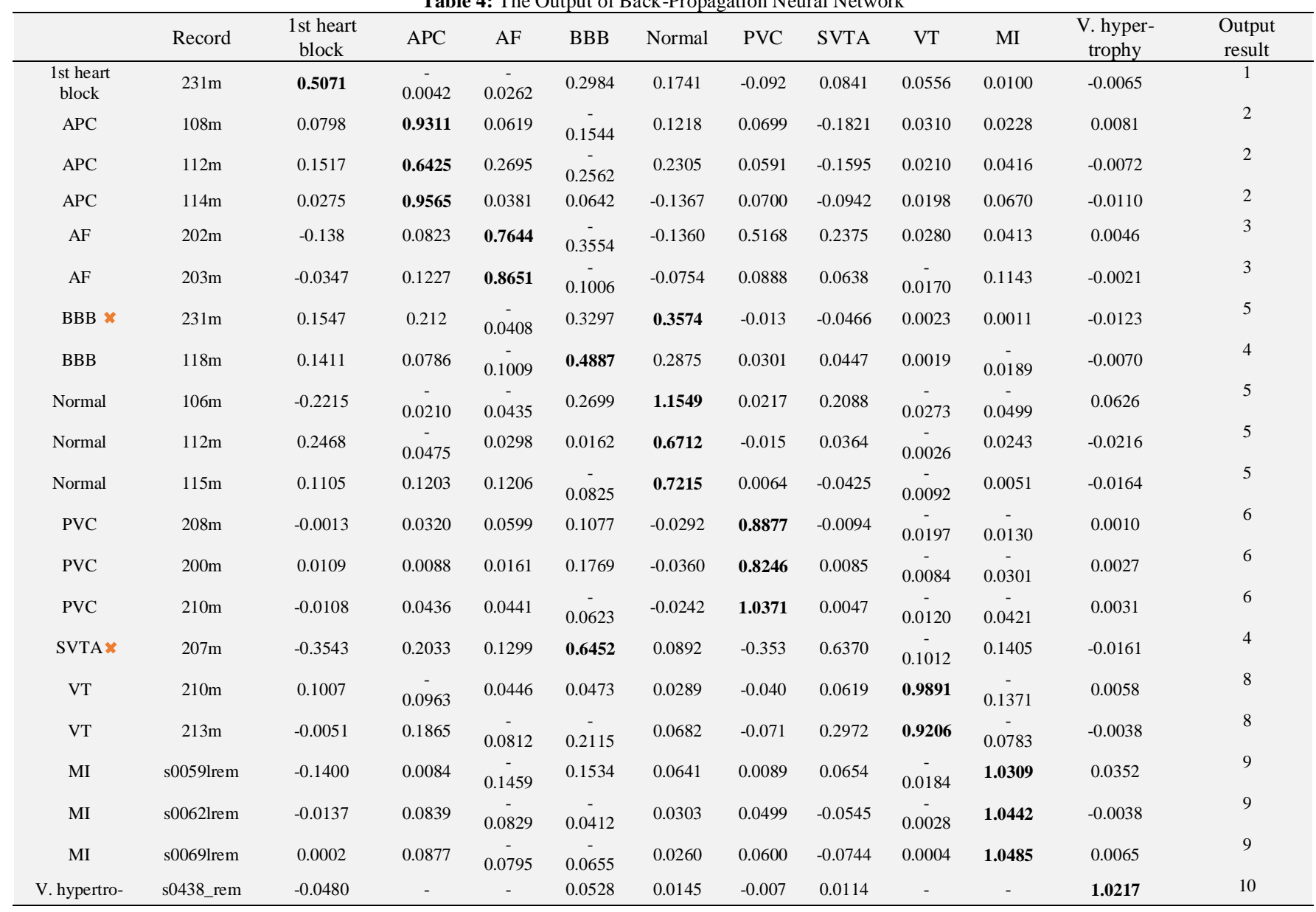




$\begin{array}{lll}\text { phy } & 0.0032 & 0.0274\end{array}$

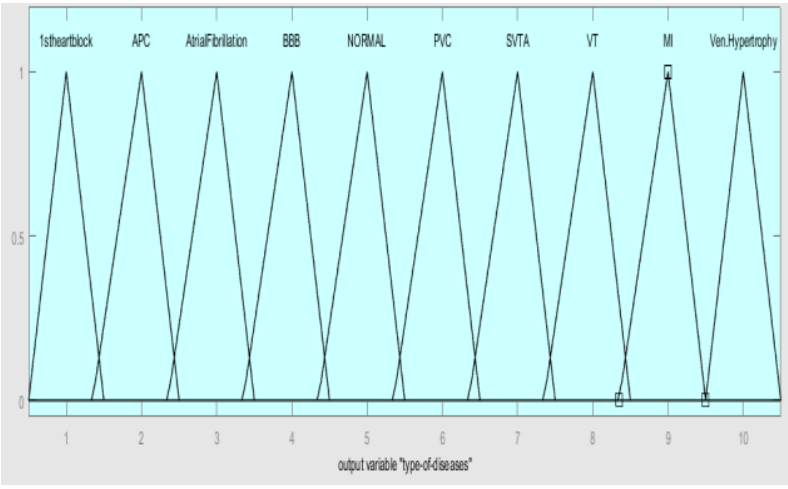

Fig. 5: The Fuzzy Output.

Table 5: The Output of Fuzzy Logic Controller.

\begin{tabular}{lll}
\multicolumn{3}{c}{ Table 5: The Output of Fuzzy Logic Controller. } \\
\hline Diseases & Record & output \\
\hline 1st heart block & $231 \mathrm{~m}$ & 1.2 \\
APC & $108 \mathrm{~m}$ & 1.95 \\
APC & $112 \mathrm{~m}$ & 1.93 \\
APC & $114 \mathrm{~m}$ & 1.95 \\
AF & $202 \mathrm{~m}$ & 2.93 \\
AF & $203 \mathrm{~m}$ & 2.94 \\
BBB & $231 \mathrm{~m}$ & 3.95 \\
BBB & $118 \mathrm{~m}$ & 3.95 \\
Normal & $106 \mathrm{~m}$ & 4.95 \\
Normal & $112 \mathrm{~m}$ & 4.95 \\
Normal & $115 \mathrm{~m}$ & 4.94 \\
PVC & $208 \mathrm{~m}$ & 5.93 \\
PVC & $200 \mathrm{~m}$ & 5.9 \\
PVC & $210 \mathrm{~m}$ & 5.94 \\
SVTA & $207 \mathrm{~m}$ & 6.95 \\
VT & $210 \mathrm{~m}$ & 7.95 \\
VT & $213 \mathrm{~m}$ & 7.95 \\
MI & s00591rem & 8.94 \\
MI & s0062lrem & 8.95 \\
MI & s0069lrem & 8.95 \\
V. hypertrophy & s0438_rem & 10 \\
\hline
\end{tabular}

\section{Conclusion}

In this paper, we built a network to classify various cardiac diseases e.g. normal, 1st-degree heart block, Atrial Premature Contraction, Atrial fibrillation, Bundle-branch block, Premature ventricular contraction, Supraventricular Tachycardia, Ventricular tachycardia, Myocardial Infraction, and Ventricle Hypertrophy. ECG signals have similar characteristics are more difficult for the network to classify accurately. A back-propagation algorithm is applied for categorization purpose that is effective. Although in our suggested work two signals are not classified because some diseases have some features similar a little and some unintentional errors, the total performance is excellent, with an accuracy of $90.4 \%$. Fuzzy inference system is used for classification purpose and shows excellent results with an accuracy is $100 \%$.

\section{References}

[1] A. A. M. A. A. B. Zahia Zidelmala, "QRS detection based on wavelet coefficients," Elsevier, 2011.

[2] B. Michael W. Zimmerman, Classification of ECG St Events as Ischemic or Non-Ischemic Using Reconstructed Phase Spaces, A Thesis submitted to the Faculty of the Graduate School, Marquette University, and May 2004.

[3] M. K. El Mimouni El Hassan *, "An FPGA-Based Implementation of a Pre-Processing Stage for ECG Signal Analysis Using DWT," in Second World Conference on Complex Systems, Agadir, Morocco, 2014 IEEE.

[4] B. N. L. M. C. D. Wai Kei Lei, "AFC-ECG: An Adaptive Fuzzy ECG Classifier," in Springer, Verlag Berlin Heidelberg, 2007.
[5] M. A. M. Taiseer Mohammed Siddig, "A Study of ECG Signal Classification using Fuzzy Logic Control," International Journal of Science and Research, vol. 3, no. 2, 2014.

[6] G. K.Amtul Salam, "An Algorithm for ECG Analysis of Arrhythmia Detection," in International Conference on Electrical, Computer and Communication Technologies, Coimbatore, India, 2015 IEEE.

[7] Y.-S. N. a. J.-Y. W. Kun-Chih (Jimmy) Chen*, "Electrocardiogram Diagnosis using Wavelet-based Artificial," in IEEE 5th Global Conference on Consumer Electronics, 2016.

[8] R. M.-M. D. L.-E. D. C. T.-A. E. R.-A. M.-P. R. C.-M. Jose Antonio Gutiérrez-Gnocchi, "DSP-based arrhythmia classification using wavelet transform and probabilistic neural network," Elsevier, no. 1746-8094, 2016.

[9] A. S. B. R. G. M. D. Sayali Tandale, "Arrhythmia Classification Using Neuro-Fuzzy Approach," IEEE, 2017.

[10] R. P. JAAKKO MAMIVUO, "Principles and Applications of Bioelectric and Biomagnetic Fields," in the Basis of ECG Diagnosis, 1995.

[11] "https://www.physionet.org/cgi-bin/atm/ATM," [Online].

[12] A. T. Hari Mohan Rai, "ECG signal classification using wavelet transform and Back Propagation Neural Network," in 5th International Conference on Computers and Devices for Communication, Kolkata, India, 2012 IEEE.

[13] M. M. H. T. B. Tanoy Debnath, "Analysis of ECG Signal and Classification of Heart Abnormalities Using Artificial Neural Network," in 9th International Conference on Electrical and Computer Engineering, Dhaka, Bangladesh, 2016 IEEE.

[14] D. N. S. S. N. D. G. Abishek Santhosh Raj A, "Auto Analysis of ECG Signals Using Artificial Neural Network," in International Conference on Science, Engineering and Management Research, Chennai, India, 2014 IEEE.

[15] D. K. P. I. A. B. K. K. Remya R S, "Classification of Myocardial Infarction Using Multi-Resolution Wavelet Analysis of ECG," in International Conference on Emerging Trends in Engineering, Science and Technology, 2015 Elsevier.

[16] V. K. G. Mayank Kumar Gautam, "A Neural Network approach and Wavelet analysis for ECG classification," in second IEEE International Conference on Engineering and Technology, Coimbatore, TN, India. 2016 IEEE.

[17] M. S. Uvais Qidwai, "Fuzzy Detection of Critical Cardiac Abnormalities using ECG data: A ubiquitous approach," in 11th International Conference on Hybrid Intelligent Systems, Melacca, Malaysia, 2011 IEEE. 